\title{
Autonomous Visualization
}

\author{
Khalid El-Arini, Andrew W. Moore, and Ting Liu \\ School of Computer Science \\ Carnegie Mellon University \\ Pittsburgh, PA 15213 USA \\ $\{k b e$, awm, tingliu\}@cs.cmu.edu
}

\begin{abstract}
Many classification algorithms suffer from a lack of human interpretability. Using such classifiers to solve real world problems often requires blind faith in the given model. In this paper we present a novel approach to classification that takes into account interpretability and visualization of the results. We attempt to efficiently discover the most relevant snapshot of the data, in the form of a two-dimensional scatter plot with easily understandable axes. We then use this plot as the basis for a classification algorithm. Furthermore, we investigate the trade-off between classification accuracy and interpretability by comparing the performance of our classifier on real data with that of several traditional classifiers. Upon evaluating our algorithm on a wide range of canonical data sets we find that, in most cases, it is possible to obtain additional interpretability with little or no loss in classification accuracy.
\end{abstract}

\section{Introduction}

In this paper we present a classification algorithm that takes into account human interpretability of the results. We attempt to find the most relevant snapshot of a data set, in the form of a two-dimensional scatter plot with easily understandable axes. This search procedure results in a transformation of our original attributes into two new features, which not only results in a potentially useful visualization of the data, but can be used as the basis for a simple classifier. Using this classifier, we can begin to investigate the trade-off between classification accuracy and interpretability. We call this process Autonomous Visualization (AV).

Previously, there has been much work on the visualization of large data sets, which usually involves projecting several dimensions onto a two-dimensional plot that is easy for humans to comprehend. de Oliveira and Levkowitz provide a recent survey of the field 4 .

Many have tackled the problem of data-driven scientific discovery 11,6]. Others have attempted to add interpretability to clustering [15141]. Our work differs from these in that we are dealing with the problem of classification, and that we do not limit our techniques to any specific application area.

Goldberger et al. describe a dimensionality reduction technique that can be used for producing visualizations of high-dimensional data 9. While their approach of finding an optimal transformation of the data to a lower-dimensional 

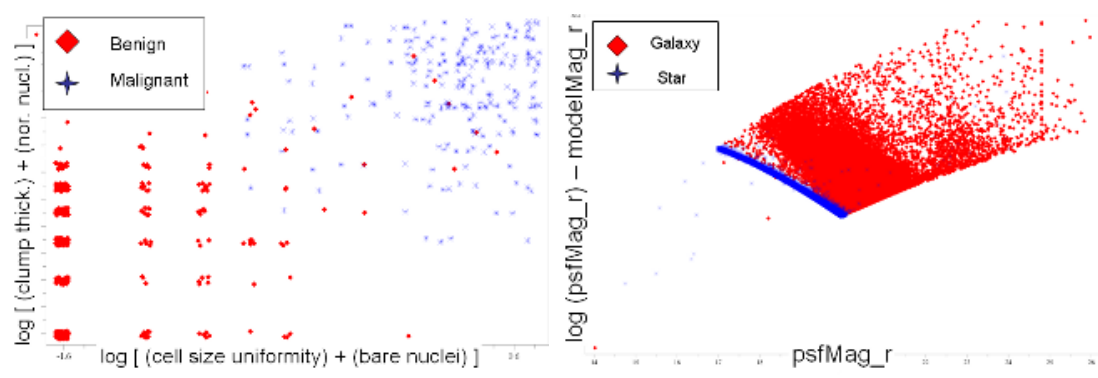

Fig. 1. Left: The AV plot for a breast cancer data set separates the benign and malignant cases along a diagonal decision boundary. Right: This SDSS star/galaxy plot produced by AV clearly separates the two classes while maintaining interpretable axes.

space before running nearest neighbor is similar in nature to what we are attempting, one key difference is that the scatter plots they produce do not have easily interpretable axes. This is also true for other techniques that produce features that are linear combinations of the inputs, such as principal components analysis and projection pursuit [7]13].

Others have proposed classifiers that take interpretability into account, but we differ by not relying on the format of a rule set or nearest neighbor to make our classifier interpretable, but rather on direct visualization [16]10]3.

\section{Methodology}

Our approach in designing a visualizable, interpretable classification algorithm assumes that the data consists of real-valued input attributes and a single, discrete output. At a high level, our technique consists of three steps: (1) We search over two-dimensional scatter plots of the data, and select the most relevant plot (where relevance in this context is defined below); (2) Given the most relevant plot, we transform the data into the two dimensions defined by its axes, and then train a simple classifier in this transformed space; (3) We classify future data points by transforming them into this two-dimensional space, and applying the classifier trained in the previous step.

\subsection{Scatter Plots}

In order to find the most relevant snapshot of the data, we search through the space of possible two-dimensional scatter plots. To ensure that the features plotted on the two axes are understandable to humans, as well as to make our search tractable, we limit the types of scatter plots we consider. First, each of the two axes in a scatter plot represents an arithmetic expression of only one or two input attributes. Expressions of more than two attributes begin losing their ease of interpretability. Furthermore, we limit the possible arithmetic expressions to ones that contain commonly understood operators. We refer to the pair of expressions that define a scatter plot as a pairexp (e.g., the axes in Figure 1). 


\section{$2.2 \quad$ Relevance}

The single most important aspect of the Autonomous Visualization process is determining which scatter plots are better than others. This relies on defining a relevance metric that allows us to score pairs of expressions on how likely they are to produce interesting visualizations of the data. One characteristic of visually appealing scatter plots is that points from the same class tend to be grouped together, and apart from points in other classes. We developed a metric based on this intuition, as well as an efficient algorithm to compute these scores.

We consider the input data to have been generated by a set of two-dimensional Gaussian distributions, one per class. Thus, we transform the data into the two dimensions defined by the current pairexp, and compute for each class $k$ the maximum likelihood Gaussian, with mean $\mu_{k}$ and covariance $\boldsymbol{\Sigma}_{k}$. We then compute the number of misclassifications that would occur if we used the Gaussians to classify the points in our data set. We define the score of the current pairexp to be this training set error. Intuitively, a plot that has well separated classes should obtain a lower training set error, and thus a better score, than a plot whose classes are not well separated. We use training set error rather than validation set error on a held out set because it is precisely the points in the training set that we wish to visualize.

Our score can now be written as $\sum_{i} I\left(c_{i} \neq \hat{c_{i}}\right)$, where $I(\cdot)$ is the indicator function, $\hat{c_{i}}=\operatorname{argmax}_{k} P\left(\mathbf{x}_{\mathbf{i}} \mid c_{i}=k\right) P\left(c_{i}=k\right)$ is the class predicted by the Gaussian Bayes classifier, and $c_{i}$ is the correct output class for point $\mathbf{x}_{\mathbf{i}}$.

\subsection{Classification}

Once we find the best pair of expressions, we have two human interpretable axes on which to plot our data. However, we also now have transformed the original $m$-dimensional input space to a two-dimensional space that can be used for Gaussian Bayes classification. We classify new data points using the maximum likelihood Gaussians learned for this new transformed space. Specifically, we compute the predicted class $\hat{c_{i}}$ for each point to be classified, as above.

\section{Acceleration}

\subsection{Accelerating Score Computation}

Naïvely, we can compute the relevance score for a pairexp by iterating through every point in the data set and checking its Gaussian Bayes classification against the true class label. This approach clearly takes linear time in the number of data points, per pairexp. However, by exploiting the spatial structure of our data, we developed an efficient algorithm for computing the score using $k d$-trees [2].

The first step in efficiently computing the score for a given pairexp of up to four attributes (i.e., up to two per expression) is constructing a $k d$-tree with these attributes as its dimensions. Note that although $k d$-tree construction is an $O(n \log n)$ operation, since we are constructing the $k d$-tree with the raw attribute 
values (i.e., no expression-specific information) we only need to construct one tree for all possible pairexps of these four attributes. With our current set of legal expressions, this amounts to one tree constructed per nearly 3,000 pairexps.

We traverse the tree linearly, and at a given $k d$-tree node, we attempt to calculate the number of misclassified points belonging to that node without actually iterating through all of them. Given the bounding hyperrectangle for the node, we check to see if any of the class Gaussians are dominant. In other words, for every geometric location $\mathbf{x}$ in the bounding box, does there exist a class $k$ such that $P\left(c_{\mathrm{x}}=k \mid \mathbf{x}\right)>P\left(c_{\mathrm{x}}=l \mid \mathbf{x}\right)$ for all classes $l$ ? If so, we say that class $k$ dominates this node, since every point will be classified as class $k$. We can thus prune our search, because all points of other classes belonging to this node will be misclassified, and we can immediately compute the score. If no class dominates the node, we sum the results of recursive calls to the node's two children. We only ever iterate through the individual points of a node if we are at a leaf node that is not dominated by any single class. For more details, please refer to our technical report [5].

Finally, it is important to note that due to the monotonicity of our scoring metric, we can perform early termination pruning, which is a significant source of computational gain. As we traverse the $k d$-tree, we can terminate immediately after computing a misclassification score that is greater than the best one so far, even if we have only looked at a tiny portion of the tree. This allows this algorithm to run much faster than the naïve one in practice.

\subsection{Accelerating Pairexp Search}

Finding the optimal scatter plot that represents the best scoring pairexp involves a hefty combinatorial search. The search can be divided into a two-stage hierarchy. At an outer level, we iterate over tuples of attributes. In an inner loop, given a tuple of attributes, we iterate over possible pairexps that can be generated from those attributes. For high dimensional data sets, it is computationally infeasible for both stages to be exhaustive, since we would have to consider $\mathrm{O}\left(\mathrm{m}^{4}\right)$ tuples, where $m$ is the number of dimensions.

Rather than exhaustively considering all possible 2-,3-, and 4-tuples of the input attributes, we instead perform a greedy search, followed by one step of hillclimbing: (1) We exhaustively consider all pairs of attributes, remembering which pair produced the best scoring pairexp (e.g., if the best scoring two attribute pairexp was $[x, \log y]$, then we remember $(x, y)$ as the best pair); (2) Given the best pair, we consider all triples that contain the best pair; (3) Given the best triple, we consider all quadruples that contain the best triple; (4) Starting from the best quadruple, we consider all other quadruples that can be obtained by changing each of the four attributes, one at a time.

Note that only the outer stage is greedy; our inner loop search over pairexps of a given tuple is exhaustive. This heuristic search now only has a quadratic dependency on $m$, since steps 2-4 are now linear. Furthermore, this quadratic dependency is ameliorated by the fact that, in contrast to quadruples, the number of pairexps that can be generated from a pair of attributes is quite limited. 


\section{Experimental Results}

We evaluated both the naïve and $k d$-tree based implementations on twelve different data sets. Eleven come from the UC Irvine repository, while EDSGC is a subset of the Edinburgh/Durham Southern Galaxy Catalogue.

\subsection{Interpretability}

We produced a scatter plot for each of the twelve data sets. Figure 1 contains a representative sample, and the entire set of plots is available in our technical report [5. We see that our algorithm succeeds in producing some separation of the classes. Furthermore, the axes represent simple expressions involving attributes that are well known to the domain experts that would be interested in analyzing the data. This stands in contrast to the alternative of plotting the data as projected onto its top two principal components, since in this case, the axes are no longer directly interpretable.

In order to verify that our algorithm was producing interesting and interpretable visualizations of the data, we consulted a domain expert in astronomy, who provided us with real data from the Sloan Digital Sky Survey (SDSS). Given ten real input attributes, and a 50,000 record subset of the data corresponding to a region of the sky deemed interesting by the astronomer, AV produced the star/galaxy plot seen in Figure 1. The domain expert confirmed that the plot was precisely the kind of plot he would expect from this data. This anecdotal evidence supports our claim that AV indeed produces useful visualizations.

Furthermore, in order to determine whether the complexity of our binary arithmetic expressions was warranted, we ran a version of our algorithm that considered only pairexps with unary expressions on the two axes. When applied to the SDSS data, this version produces a rather uninspiring plot, indicating that there are indeed data sets that are best visualized by combining multiple attributes on a single axis.

\subsection{Classification Accuracy}

We computed five-fold cross validation accuracies after running our AV classifier on all the data sets. For the sake of comparison, we ran nine canonical classifiers on the same data [17. It should be noted that, for the sake of expediency, we limited all larger data sets to 5,000 randomly selected records.

When we compare the classification accuracies of our classifier with those of the canonical algorithms, we find that the AV classifier is competitive. In Figure2, we see a pairwise comparison between our classifier and the nine canonical classifiers. On average, the classification accuracy of AV is only 0.168 percentage points lower than the other algorithms. In fact, AV outperforms 1-nearest neighbor and naïve Bayes. On these data sets, the best performing algorithm was a support vector machine with quadratic kernels, and AV is only 2.348 percentage 

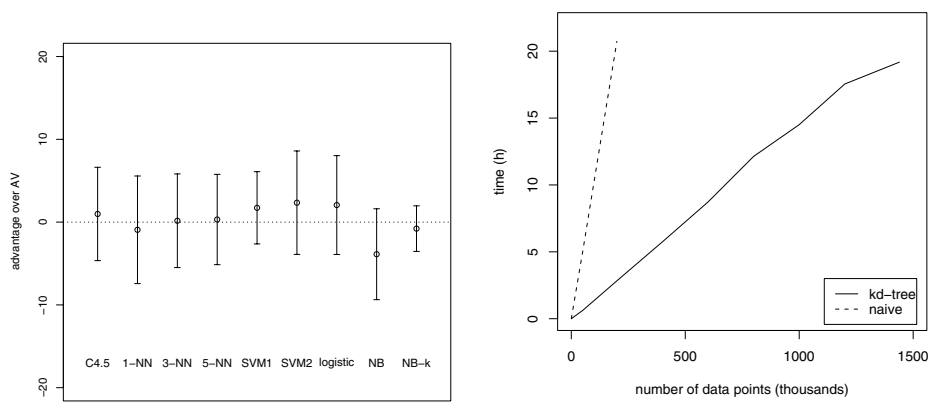

Fig. 2. Left: A pairwise comparison between AV and canonical classifiers, showing the difference in classification accuracy, averaged over all data sets. Right: Timing analysis of AV on expanded EDSGC data set, as number of records is increased.

points less accurate on average. In Table 1, we see that AV outperforms the average canonical classifier on all data sets except iris, realmpg, sonar and vehicle. However, although close at times, AV is never the most accurate classifier on a given data set.

\subsection{Efficiency}

Table 1 shows timing results for running AV with both the $k d$-tree and non$k d$-tree implementations of the algorithm. We see that by implementing our algorithm using $k d$-trees, we make noticeable gains in efficiency over the naïve implementation, especially for larger data sets. The adult data set, for instance, sees a 4.12 fold speed-up, and the EDSGC data set sees a speed-up of 8.12. It is important to note, however, that the vehicle data set performs slightly more slowly under the $k d$-tree algorithm than the naïve one. This is perhaps due to the relatively small size of the data set preventing the algorithm from overcoming the six pairwise class comparisons it must do per $k d$-tree node, a problem not encountered in the 50,000 record forest data set, despite its seven output classes.

Furthermore, we timed the $k d$-tree implementation on the EDSGC data set as we varied the number of points from 50,000 to the full 1.4 million record data set. The resulting plot, as shown in Figure 2, is mostly linear, but begins to dip slightly as we approach the size of the full data set. When compared to the plot for the naïve implementation, we see that the overhead of building the $k d$-trees is a small price to pay for the gain in efficiency.

It is important to note that the timing costs reported here are only incurred during the training phase of the classifier. Once a relevant pairexp has been found during training, future data points can be classified simply by transforming them to the two-dimensional space and applying the Gaussian Bayes classifier. Alternatively, for a large data set, time can be saved by training only on a subset 
Table 1. Classification accuracy and timing results

\begin{tabular}{rcc|ccc|cc} 
& & & \multicolumn{3}{|c|}{$\begin{array}{c}\text { Accuracy } \\
\text { Data set Records Attributes }\end{array}$} & \multicolumn{2}{c}{ Timing (sec) } \\
\cline { 5 - 7 } Abalone & 4178 & 8 & 55.47 & 53.28 & 53.84 & 308 & 339 \\
Adult & 48844 & 6 & 83.92 & 80.87 & 82.02 & 502 & 2069 \\
Breast-w & 701 & 9 & 97.42 & 96.15 & 96.19 & 22 & 59 \\
Diabetes & 770 & 8 & 77.47 & 74.51 & 77.34 & 22 & 55 \\
EDSGC & 50000 & 22 & 99.46 & 95.16 & 99.16 & 2221 & 18037 \\
Forest & 50000 & 10 & 69.82 & 67.40 & 68.12 & 4856 & 8445 \\
Heart-statlog & 272 & 13 & 85.19 & 80.16 & 81.48 & 21 & 46 \\
Ionosphere & 357 & 34 & 91.74 & 87.59 & 91.17 & 69 & 183 \\
Iris & 155 & 4 & 96.67 & 95.78 & 93.33 & 2.0 & 7.2 \\
Realmpg & 394 & 7 & 82.91 & 72.79 & 70.66 & 2.0 & 6.8 \\
Sonar & 210 & 60 & 87.02 & 77.51 & 74.04 & 140 & 227 \\
Vehicle & 851 & 18 & 80.50 & 69.35 & 60.52 & 279 & 268 \\
\hline
\end{tabular}

of the data in order to obtain the best pairexp, but then transforming the entire data set for visualization purposes.

\section{Discussion}

There is much flexibility inherent to the AV process, which provides ample opportunity for exploration during algorithm design. For instance, before settling on the Gaussian Bayes misclassification score, we implemented several others for comparison. The first attempt was a pairwise count that penalized points of differing classes that were in close proximity to each other. We found that this algorithm generally produced poorer visualizations, and relied on several parameters that we found difficult to set. (Leban et al. propose a similar method, which they use to successfully analyze and visualize gene expression data [12.) In another attempt, we tried maximizing the Gaussian log likelihood of the data, and found that while we produced visualizations that were at least as good as our current results, it was difficult to optimize the algorithm in terms of efficiency, which we believe is the key to making it tractable to search such an intense diversity of scatter plots. We faced a similar situation when we tried a Gaussian misclassification score that allowed full covariance matrices, rather than the diagonal matrices that we use in our current algorithm. In fact, since our relevance score is simply a training set error, conceivably any classifier can be substituted for the Gaussian Bayes classifier that we use, either for computing the score or for classifying new data.

\section{Conclusion}

We have described a novel approach to classification that takes into account interpretability of the results. We have detailed an algorithm that produces rel- 
evant scatter plots of real-valued data sets, and evaluated it in terms of interpretability, classification accuracy, and efficiency. For most problems we considered, our algorithm was competitive with state-of-the-art classifiers. We provided compelling evidence that it is possible to obtain additional interpretability with little or no loss in classification accuracy. Furthermore, we showed that our algorithm is efficient, making it feasible for use on large, real-world data sets. Finally, our results demonstrate the potential for further investigation into the opportunities and challenges of integrating visualization with classification.

\section{References}

1. R. Agrawal, J. Gehrke, D. Gunopulos, and P. Raghavan. Automatic subspace clustering of high dimensional data for data mining applications. In Proc. ACM SIGMOD International Conference on Management of Data, pages 94-105, 1998.

2. J. Bentley. Multidimensional binary search trees used for associative searching. Commun. ACM, 18(9):509-517, 1975.

3. R. Caruana, H. Kangarloo, J. David, N. Dionisio, U. Sinha, and D. Johnson. Casebased explanation of non-case-based learning methods. In Proc. American Medical Informatics Association Symposium, pages 212-215, 1999.

4. M.C.F. de Oliveira and H. Levkowitz. From visual data exploration to visual data mining: A survey. IEEE Trans. Visualization and Computer Graphics, 9(3), July-September 2003.

5. K. El-Arini, A. W. Moore, and T. Liu. Autonomous visualization. Technical Report CMU-CS-06-137, Carnegie Mellon University, 2006.

6. B. C. Falkenhainer and R. S. Michalski. Integrating quantitative and qualitative discovery: The ABACUS system. Machine Learning, 1(4):367-401, 1986.

7. J. H. Friedman and J. W. Tukey. A projection pursuit algorithm for exploratory data analysis. IEEE Trans. Computers, C-23(9):881-889, 1974.

8. I. Galkin, B. Reinisch, X. Huang, R. Benson, and S. Fung. Automated diagnostics for resonance signature recognition on IMAGE/RPI plasmagrams. Rad. Sci., 2004.

9. J. Goldberger, S. Roweis, G. Hinton, and R. Salakhutdinov. Neighbourhood components analysis. In Advances in Neural Information Processing Systems. 2005.

10. R. C. Holte. Very simple classification rules perform well on most commonly used datasets. Machine Learning, 11(1):63-90, 1993.

11. P. Langley. Data-driven discovery of physical laws. Cog. Science, 5(1):31-54, 1981.

12. G. Leban, M. Mramor, I. Bratko, and B. Zupan. Simple and effective visual models for gene expression cancer diagnostics. In Proc. ACM SIGKDD International Conference on Knowledge Discovery in Data Mining, pages 167-176, 2005.

13. E. Lee, D. Cook, S. Klinke, and T. Lumley. Projection pursuit for exploratory supervised classification. Comp. and Graphical Statistics, 14(4):831-846, 2005.

14. D. Pelleg and A.W. Moore. Mixtures of rectangles: Interpretable soft clustering. In Proc. 18th International Conference on Machine Learning, pages 401-408, 2001.

15. T. Sprenger, R. Brunella, and M. Gross. H-BLOB: A hierarchical visual clustering method using implicit surfaces. In Proc. Visualization '00, pages 61-68, 2000.

16. S. M. Weiss, R. S. Galen, and P. V. Tadepalli. Maximizing the predictive value of production rules. Artificial Intelligence, 45(1-2):47-71, 1990.

17. I. H. Witten and E. Frank. Data Mining: Practical machine learning tools with Java implementations. Morgan Kaufmann, San Francisco, CA, 2000. 